\title{
TITLE:
}

\section{Kernel method for corrections to scaling}

$\operatorname{AUTHOR}(\mathrm{S})$ :

Harada, Kenji

\section{CITATION:}

Harada, Kenji. Kernel method for corrections to scaling. Physical Review E 2015, 92(1): 012106.

ISSUE DATE:

2015-07-06

URL:

http://hdl.handle.net/2433/198679

RIGHT:

(C2015 American Physical Society 
PHYSICAL REVIEW E 92, 012106 (2015)

\title{
Kernel method for corrections to scaling
}

\author{
Kenji Harada \\ Graduate School of Informatics, Kyoto University, Kyoto 606-8501, Japan \\ (Received 9 October 2014; revised manuscript received 7 May 2015; published 6 July 2015)
}

\begin{abstract}
Scaling analysis, in which one infers scaling exponents and a scaling function in a scaling law from given data, is a powerful tool for determining universal properties of critical phenomena in many fields of science. However, there are corrections to scaling in many cases, and then the inference problem becomes ill-posed by an uncontrollable irrelevant scaling variable. We propose a new kernel method based on Gaussian process regression to fix this problem generally. We test the performance of the new kernel method for some example cases. In all cases, when the precision of the example data increases, inference results of the new kernel method correctly converge. Because there is no limitation in the new kernel method for the scaling function even with corrections to scaling, unlike in the conventional method, the new kernel method can be widely applied to real data in critical phenomena.
\end{abstract}

DOI: 10.1103/PhysRevE.92.012106

PACS number(s): 05.70.Jk, 02.50.Tt

\section{INTRODUCTION}

Critical phenomena relate many fields of science. Because of a scale invariant at a critical point, a scaling law exists and different systems share a set of values of scaling exponents. This is a universality of critical phenomena. The set of exponent values defines a universality class of critical phenomena. The classification of various critical phenomena has been extensively studied from the viewpoint of universality. Scaling laws exist not only in the thermodynamic limit but also in finite-size systems. In particular, by using a finite-size scaling law for finite-size systems, we have confirmed various universality classes [1]. However, such studies have often suffered from corrections to scaling. For example, in the study of spin glass transition, we often experience difficulties attributed to strong corrections to scaling, because the size of the system calculated is very limited. In the study of exotic quantum criticality (e.g., the deconfined quantum criticality [2], which is beyond the Landau-Ginzburg-Wilson paradigm), the existence of corrections to scaling increases the difficulty in distinguishing exotic quantum criticality from a weak firstorder transition [3-10]. Corrections to scaling are caused by the existence of irrelevant scaling variables, which is generally predicted by renormalization-group theory. Therefore, treating corrections to scaling is an important issue in research on critical phenomena.

The purpose of this work is to present a new approach to treat corrections to scaling. With or without corrections to scaling, the most conventional method in the scaling analysis of critical phenomena is the least-squares method. It is based on chi-square statistics to infer scaling exponents and a scaling function from data. If we propose a certain model function for the scaling, the least-squares method has no limitation. However, because we usually do not know the specific scaling function, we assume a polynomial as the model function in the least-squares method (see Ref. [11]). Unfortunately, since it is difficult to set the degree of the polynomial so that it approximates the scaling function in the range of data points, we have to limit the range of data to a narrow region near a critical point (see Fig. 4 in Ref. [12]). The Bayesian inference method [12] was recently proposed to resolve this inconvenient problem; it is based on a kernel method as the Gaussian process regression. Recent studies of various critical phenomena have proved the effectiveness of the kernel method [9,10,13-19]. In this work, we extend the Bayesian inference method to the problem of corrections to scaling. The new kernel method is flexible even with corrections to scaling, because only the smoothness of the scaling function related to relevant scaling variables is assumed. Thus, it can be a promising tool for studying critical phenomena whose data cannot be treated by using the conventional approach.

The remaining parts of this paper are organized as follows. In Sec. II, we will give a brief introduction to corrections to scaling in the scaling analysis. In Sec. III, we will first introduce the Bayesian inference and the Gaussian process regression for the scaling analysis. We will then consider the ill-posedness of an inference problem in the scaling analysis with corrections to scaling, and we will propose a new composite kernel method. In Sec. IV, we will explain the details of the practical procedure for the new kernel method. After that, we will report the performance of the new kernel method for example data sets in detail. In Sec. V, we will summarize this work.

\section{SCALING ANALYSIS AND ILL-POSEDNESS BY CORRECTIONS TO SCALING}

In this paper, for the sake of simplicity, we consider a finite-size scaling law with a relevant scaling variable and an irrelevant one. Although the irrelevant scaling variable vanishes in the thermodynamic limit, it has an effect in a finite-size region, causing a correction to scaling in the finite-size scaling law as

$$
A(t, u, L)=L^{c_{2}} F\left[t L^{c_{1}}, u L^{-c_{3}}\right],
$$

where $L$ is the size of a system, and a scaling variable $t(u)$ is relevant (irrelevant). Thus, $c_{1}$ and $c_{3}$ are positive. The universality class of critical phenomena is defined by the exponent values of the relevant scaling variables. The scaling function in the thermodynamic limit is $F[\cdot, 0]$, and the correction to scaling is $F\left[t L^{c_{1}}, u L^{-c_{3}}\right]-F\left[t L^{c_{1}}, 0\right]$.

The object of the scaling analysis is to determine the critical exponents from a data set of $A(t, u, L)$ by using the finite-size 
scaling law, Eq. (1). Using the new rescaled variables,

$$
X_{1} \equiv t L^{c_{1}}, X_{2} \equiv u L^{-c_{3}}, Y \equiv A / L^{c_{2}},
$$

we can rewrite Eq. (1) as

$$
Y=F\left(X_{1}, X_{2}\right) \pm E,
$$

where $E \equiv \delta A / L^{c_{2}}$ and $\delta A$ is the data precision of $A$. Thus, the scaling analysis is a statistical inference of critical exponents so that rescaled data points collapse on a surface $F$ with precision $E$.

In general, we can know the value of a relevant scaling variable $t$. For example, $t \equiv T-T_{c}$, where $T$ is a temperature and $T_{c}$ is a critical temperature. However, we do not know that of an irrelevant scaling variable $u$. If $u$ is constant and nonzero, the right-hand side of Eq. (1) can be rewritten as $L^{c_{2}} F\left[t L^{c_{1}}, u L^{-c_{3}}\right]=L^{c_{2}} G\left[t L^{c_{1}}, L\right]=H[t, L]$. Finally, the trivial scaling function can be defined as $H$. Therefore, the inference problem in the scaling analysis with a correction to scaling is ill-posed.

\section{BAYESIAN INFERENCE FOR SCALING ANALYSIS}

\section{A. Bayesian inference}

If there is no irrelevant scaling variable, the definition of scaling function $F\left[X_{1}, 0\right]$ has no ambiguity. We can safely apply a statistical inference method to the scaling analysis. The most popular method is based on the least-squares method. From Eq. (3), we can assume that the difference between $Y$ and $F\left[X_{1}, 0\right]$ obeys the Gaussian distribution with mean 0 and variance $E^{2}$. Thus,

$$
P(Y)=\frac{1}{\sqrt{2 \pi E^{2}}} \exp \left(-\frac{\left(Y-F\left[X_{1}\right]\right)^{2}}{2 E^{2}}\right),
$$

where $F[X] \equiv F[X, 0], Y=A(T, L) / L^{c_{2}}, X_{1}=\left(T-T_{c}\right) L^{c_{1}}$, $E=\delta A(T, L) / L^{c_{2}}$. The joint probability distribution of all points $\vec{Y} \equiv[Y(1), Y(2), \cdots]^{t}$ is written as

$$
\log P(\vec{Y})=-\sum_{i} \frac{\left\{Y(i)-F\left[X_{1}(i)\right]\right\}^{2}}{2 E(i)^{2}}-\sum_{i} \frac{\log \left[2 \pi E(i)^{2}\right]}{2} .
$$

In the least-squares method, we assume the explicit form of the scaling function $F[x]$ as a parametric function with a parameter set $\vec{a} \equiv\left(a_{0}, a_{1}, \cdots\right)^{t}$. The best value of $\vec{a}$ is inferred by maximizing the first term in the right hand of Eq. (5), because the second term does not depend on $\vec{a}$.

From the view point of Bayesian inference, this procedure can be derived as follows. The right-hand side of Eq. (5) depends on the parameter set $\vec{a}$. Thus, it can be regarded as a conditional probability of $\vec{Y}$ for $\vec{a}$ as $P(\vec{Y} \mid \vec{a})$. According to Bayes' theorem, a conditional probability of $\vec{a}$ for $\vec{Y}$ can be written as

$$
P(\vec{a} \mid \vec{Y})=\frac{P(\vec{Y} \mid \vec{a}) P(\vec{a})}{P(\vec{Y})} .
$$

When we do not know a prior distribution $P(\vec{a})$, we usually suppose it as uniform. Then,

$$
P(\vec{a} \mid \vec{Y}) \propto P(\vec{Y} \mid \vec{a}) .
$$

Therefore, we can infer the most probable value of $\vec{a}$ by simply maximizing the right-hand side of Eq. (5) in Bayesian inference. It is called maximum a posteriori probability (MAP) estimation in the field of Bayesian inference. The least-squares method is a MAP estimation with Eq. (5).

In many cases, a regression function $F[\cdot]$ linearly depends on parameters in $\vec{a}$ : for example, polynomial. Then, we can use a simple $\chi^{2}$ test to check the quality of fit. However, $F\left[X_{1}\right]$ also includes physical parameters as $\left(T_{c}, c 1, c 2\right)$ nonlinearly. Thus, we cannot simply apply a $\chi^{2}$ test to check the quality of fit with physical parameters.

We notice that the Bayesian inference does not restrict the form of a conditional probability as Eq. (5). Therefore, we can design a suitable conditional probability for the scaling analysis as follows.

\section{B. Gaussian process regression}

As mentioned above, we often use the least-squares method for a scaling analysis. However, because one has to assume the form of the unknown scaling function as like a polynomial, this sets limits on the data in a narrow region near a critical point. To resolve this difficulty, we introduced the view point of Bayesian inference in a scaling analysis in Ref. [12]. In particular, we explored the use of Gaussian process regression in a scaling analysis. Gaussian process regression relies on a kernel function that defines the covariance of the data, so it is called a kernel method. Regarding data points as a Gaussian process with a covariance matrix $\Sigma$, the conditional probability in Eq. (5) is changed to

$$
P(\vec{Y} \mid \vec{a})=\frac{1}{\sqrt{|2 \pi \Sigma|}} \exp \left(-\frac{\vec{Y}^{t} \Sigma^{-1} \vec{Y}}{2}\right),
$$

where $\vec{a}$ is a parameter set and $(\Sigma)_{i j} \equiv k(i, j)$. The kernel function $k(\cdot, \cdot)$ ensures that $\Sigma$ is positive definite. If we use a Gaussian kernel $k_{G}$, the smoothness of the regression function, which is a scaling function in our case can be represented by a few parameters. The Gaussian kernel function in Ref. [12] is written as

$k_{G}(i, j) \equiv\left(E(i)^{2}+\theta_{2}^{2}\right) \delta_{i j}+\theta_{0}^{2} \exp \left\{-\frac{\left|X_{1}(i)-X_{1}(j)\right|^{2}}{2 \theta_{1}^{2}}\right\}$.

Here, we introduce new hyper parameters as $\left(\theta_{0}, \theta_{1}, \theta_{2}\right)$. If $X_{1}(i)$ is far from $X_{1}(j)$, the kernel function exponentially decays. The characteristic length scale is controlled by a parameter $\theta_{1}$. Since the covariance $(\Sigma)_{i j}$ represents a correlation between $Y(i)$ and $Y(j)$, the parameter $\theta_{1}$ represents the local smoothness of a scaling function. The Gaussian kernel function only restricts the local smoothness of the scaling function. It does not restrict the global shape of the scaling function as in the least-squares method. Therefore, no limitation on the range of data is needed in the kernel method. We notice that the number of hyperparameters is only 3 as $\left(\theta_{0}, \theta_{1}, \theta_{2}\right)$. For the least-squares method, we probably need many parameters in a regression function to fit data in a wide range.

In practice, we can infer the best values of parameters $\vec{a}=\left(T_{c}, c_{1}, c_{2}, \theta_{0}, \theta_{1}, \theta_{2}\right)^{t}$ by maximizing the likelihood [the right-hand side of Eq. (8) with Eq. (9)]. We used the 
conventional nonlinear optimization method to find a maximum point. In such algorithms, we need the derivative of Eq. (8) for a parameter $a$. Then, we can use the following formula:

$$
\begin{aligned}
\frac{\partial \log P(\vec{Y} \mid \vec{a})}{\partial a}= & -\frac{1}{2} \operatorname{Tr}\left(\Sigma^{-1} \frac{\partial \Sigma}{\partial a}\right) \\
& -\vec{Y}^{t} \Sigma^{-1} \frac{\partial \vec{Y}}{\partial a}+\frac{1}{2} \vec{Y}^{t} \Sigma^{-1} \frac{\partial \Sigma}{\partial a} \Sigma^{-1} \vec{Y} .
\end{aligned}
$$

Combining Eq. (7) with Eq. (8), we can define the distribution function of parameters under given data points. If we use a Monte Carlo method with the weight as Eq. (8), we can evaluate an average and a confidential interval of a parameter under given data points.

In the Gaussian process regression, we can also infer the $Y$ value of a new additional point $(X, Y)$. In fact, we assume that all data points obey a Gaussian process. In other words, the joint probability distribution of given data points and the new additional point $(X, Y)$ is also a multivariate Gaussian distribution as Eq. (8). Thus, a conditional probability of $Y$ for given data points and a parameter can be written by a Gaussian distribution with mean $\mu(X)$ and variance $\sigma^{2}(X)$ :

$$
\begin{gathered}
\mu(X) \equiv \vec{k}^{t} \Sigma^{-1} \vec{Y}, \\
\sigma^{2}(X) \equiv k(X, X)-\vec{k}^{t} \Sigma^{-1} \vec{k},
\end{gathered}
$$

where $\vec{k} \equiv\{k[X(1), X], k[X(2), X], \ldots\}^{t}$.

\section{Composite kernel}

The Gaussian kernel method can be formally generalized to the scaling analysis with an irrelevant scaling variable in Eq. (1). Because the scaling function in Eq. (1) smoothly depends on scaling variables, it can be represented by a Gaussian kernel of a two-dimensional space of $X_{1}$ and $X_{2}$. However, because of the scaling function's ambiguity as discussed above, the representation does not work. The assumption of smoothness of a scaling function does not resolve this ambiguity.

To resolve it, we consider a Taylor expansion of a scaling function $F\left[X_{1}, X_{2}\right]$ by an irrelevant rescaled variable $X_{2}$ at a point $\left(X_{1}, 0\right)$ as

$$
F\left[X_{1}, X_{2}\right]=\sum_{n=0}^{\infty} f_{n}\left(X_{1}\right)\left(X_{2}\right)^{n},
$$

where $\left.f_{n}\left(X_{1}\right) \equiv \frac{1}{n !} \frac{d^{n} F[x, y]}{d y^{n}}\right|_{\left(X_{1}, 0\right)}$ and $f_{0}(\cdot)$ is a scaling function in the thermodynamic limit. When we introduce the cutoff of the degree of the Taylor expansion, the functional form of the irrelevant rescaled variable $X_{2}$ is always a polynomial without ambiguity. In addition, the function $f_{n}$ depends only on the relevant scaling variable. Therefore, there is no illposedness in the inference problem for the scaling function. If we maintain the dependence on the relevant variable as the general functional form as $f_{n}$, then the Gaussian process regression can be defined by a composite kernel written as

$$
\begin{aligned}
k_{C}(i, j) \equiv & \delta_{i j}\left[E(i)^{2}+\theta_{2}^{2}\right] \\
& +\sum_{n=0}^{M} \theta_{n, 0}^{2} \exp \left[-\frac{\left|X_{1}(i)-X_{1}(j)\right|^{2}}{2 \theta_{n, 1}^{2}}\right]\left[X_{2}(i) X_{2}(j)\right]^{n},
\end{aligned}
$$

where $M$ is the cutoff of the Taylor expansion. Here, we introduce new hyperparameters as $\left(\theta_{n, 0}, \theta_{n, 1}\right)$ for $0 \leqslant n \leqslant M$ and $\theta_{2}$. To derive the composite kernel, we assume that variables $X_{1}$ and $X_{2}$ are statistically independent. Since the kernel function represents the covariance between two points $i$ and $j$, the total kernel is a simple product of kernels for each variable: a Gaussian kernel for $f_{n}\left(X_{1}\right)$ and a polynomial kernel for $X_{2}^{n}$. The Gaussian function part in Eq. (14) as $\theta_{n, 0}^{2} \exp \left[-\frac{\left|X_{1}(i)-X_{1}(j)\right|^{2}}{2 \theta_{n, 1}^{2}}\right]$ represents the local smoothness of $f_{n}$ without the assumption of the global shape of $f_{n}$. Therefore, it does not set any limits on the data near a critical point. We notice that the case of $M=0$ is the original kernel without a correction to scaling in Ref. [12] [Eq. (9) in this paper]. Thus, the present approach for the correction to scaling is a systematical extension of the previous kernel method of the scaling analysis.

\section{PERFORMANCE OF THE COMPOSITE KERNEL METHOD}

We will now test the performance of the composite kernel method. In this section, for the sake of simplicity, we set the cutoff of the Taylor expansion as $M=1$. We simply call the case of $M=1$ the composite kernel in this section.

At first, we will explain computational techniques for the kernel method in Secs. IV A-IV C. After that, in Secs. IV D and IVE, we will report the performance of the composite kernel method in detail.

\section{A. Normalization of rescaled variables}

In Gaussian process regression, we first use a nonlinear optimization to find a good starting point for Monte Carlo sampling. Although there is no absolute scale for rescaled variables, we found that the normalization of rescaled variables increases the numerical stability of the optimization process at the first stage of Gaussian process regression. In this work, we set the unit of length scale by the largest system size as

$$
\begin{aligned}
X_{1} & =\left(T-T_{c}\right)\left(L / L_{\max }\right)^{c_{1}} / R_{X}, \\
X_{2} & =\left(L / L_{\min }\right)^{-c_{3}}, \\
Y & =\left[A /\left(L / L_{\max }\right)^{c_{2}}-Y_{0}\right] / R_{Y}, \\
E & =\delta A /\left(L / L_{\max }\right)^{c_{2}} / R_{Y},
\end{aligned}
$$

where $L_{\max }$ and $L_{\min }$ are the largest and smallest system sizes in a data set, respectively. The values of $X_{1}$ and $Y$ and $E$ for the largest system are independent of relevant scaling exponents ( $c_{1}$ and $c_{2}$ ). The scaling factor $R_{X}$ is defined so that the width of $X_{1}$ for the largest system is 2 . The scaling factor $R_{Y}$ and the shift parameter $Y_{0}$ are defined so that $Y$ for the largest system is in $[-1: 1]$. Since data of the largest system most likely affect the inference of a scaling function, the normalization of rescaled variables may separate the inference of a scaling function from those of scaling exponents.

\section{B. Hybrid Monte Carlo sampling}

In the inference process of averages and confidential intervals of parameters, we use a hybrid Monte Carlo method (see a review in Ref. [20]) for normalized rescaled variables to construct samples of parameters by using the likelihood of 
Gaussian process regression. It is very effective for a Monte Carlo sampling of continuous variables.

We consider the weight $W(\vec{q})$ of a sampling parameter $\vec{q}$. For example, in Gaussian process regression for a scaling analysis by the composite kernel, $\vec{q}$ means inferred parameters as $\vec{a}=\left(T_{c}, c_{1}, c_{2}, c_{3}, \theta_{2}, \theta_{0,0}, \theta_{0,1}, \ldots, \theta_{M, 0}, \theta_{M, 1}\right)^{t} . W(\vec{q})$ is written as the likelihood of Eq. (8).

In the hybrid Monte Carlo sampling, we introduce an artificial momentum $\vec{p}$. Then, the artificial Hamiltonian is written as

$$
H(\vec{q}, \vec{p})=U(\vec{q})+\frac{\vec{p}^{2}}{2 m},
$$

where $U(\vec{q})=-\log W(\vec{q})$ and $m$ is an artificial mass. At first, we set an initial $\vec{p}(0)$ by a random variable, which obeys a Gaussian distribution with mean 0 and variance $m$, and $\vec{q}(0)=$ $\vec{q}$. We calculate an artificial time-evolution of $[\vec{q}(t), \vec{p}(t)]$ until an artificial time $T$ by using a leap-flog integration scheme:

$$
\begin{aligned}
& \vec{p}(t+\epsilon / 2)=\vec{p}(t)-(\epsilon / 2) \nabla U[\vec{q}(t)], \\
& \vec{q}(t+\epsilon)=\vec{q}(t)+(\epsilon / m) \vec{p}(t+\epsilon / 2), \\
& \vec{p}(t+\epsilon)=\vec{p}(t+\epsilon / 2)-(\epsilon / 2) \nabla U[\vec{q}(t+\epsilon)],
\end{aligned}
$$

where $\epsilon$ is a discrete time step. We accept $\vec{q}(T)$ as a next sample with the probability

$$
\min (1, \exp \{-H[\vec{q}(T), \vec{p}(T)]+H[\vec{q}(0), \vec{p}(0)]\}) .
$$

If not, a sample $\vec{q}$ is remained. Since the leap-flog integration conserves the total energy, the acceptance probability is almost 1. Therefore, the performance is much more effective than that of the conventional approach using random walks to make trail samples. In fact, we have to choose a suitable $m$ and $T$. In many cases, we need to introduce individual masses. The practical details are explained in Ref. [20].

\section{Practical procedure of the kernel method}

In this subsection, we summarize a practical calculation procedure in our kernel method for a scaling analysis. It is based on the weight of parameters $\vec{q}(=\vec{a})$ as

$$
W(\vec{q}) \equiv \frac{1}{\sqrt{|2 \pi \Sigma|}} \exp \left(-\frac{\vec{Y}^{t} \Sigma^{-1} \vec{Y}}{2}\right),
$$

where $(\Sigma)_{i j} \equiv k_{C}(i, j)$ and the composite kernel $k_{C}(i, j)$ is defined by Eq. (14). The inferred parameter is $\vec{q}=$ $\left(T_{c}, c_{1}, c_{2}, c_{3}, \theta_{2}, \theta_{0,0}, \theta_{0,1}, \ldots, \theta_{M, 0}, \theta_{M, 1}\right)^{t}$. In addition, we use normalized rescaled variables in Eq. (15). We estimate an average and a confidential interval of parameters from this weight under a given data set by the hybrid Monte Carlo sampling.

At first, to prepare a good starting point of Monte Carlo method, we find a maximum point of a logarithmic weight $W(\vec{q})$ by using a nonlinear optimization algorithm as the Fletcher-Reeves conjugate gradient algorithm. The derivative of a weight by $\vec{q}$ is written as

$$
\begin{aligned}
\frac{\partial \log W(\vec{q})}{\partial q_{i}}= & -\frac{1}{2} \operatorname{Tr}\left(\Sigma^{-1} \frac{\partial \Sigma}{\partial q_{i}}\right) \\
& -\vec{Y}^{t} \Sigma^{-1} \frac{\partial \vec{Y}}{\partial q_{i}}+\frac{1}{2} \vec{Y}^{t} \Sigma^{-1} \frac{\partial \Sigma}{\partial q_{i}} \Sigma^{-1} \vec{Y}
\end{aligned}
$$

The Cholesky decomposition of $\Sigma$ is useful to calculate the right-hand side of Eq. (20). We notice that the initial values of parameters are important, because a nonlinear optimization scheme generally has no global convergence. In many cases, we can guess the good initial values of physical parameters $\left(T_{c}, c_{1}, c_{2}, c_{3}\right)$. We can also prepare the initial values of hyper parameters $\left(\theta_{2}, \theta_{0,0}, \theta_{0,1}, \ldots, \theta_{M, 0}, \theta_{M, 1}\right)$, because we normalize the range of data points by introducing the normalization of rescaled variables. In fact, we always start from 1 for all hyperparameters in the following cases. The hyperparameter $\theta_{2}$ represents the total fidelity of a data set. Thus, $\theta_{2}=1$ implies that all data points has no fidelity. In fact, the values of parameters can widely move at the early stage of nonlinear optimization. It avoids trapping in local maximums. If the precision of data is enough, the inference process will finally converge with small $\theta_{2}$.

To infer averages and confidential intervals of parameters, we make many samples of parameters by the weight in Eq. (19). We recommend a hybrid Monte Carlo sampling to estimate an average and a confidential interval of $\vec{q}$. The practical details of the hybrid Monte Carlo method are reviewed in Ref. [20].

By using numerical libraries for the implementation of our kernel method, we can easily make a code of our kernel method. The performance tests in the following cases have been done by a reference $\mathrm{C}++$ code of our kernel method [21].

\section{Artificial data sets}

\section{Dimensionless observable}

We first apply the composite and original kernel methods to two artificial data sets with different types of correction forms. The first data are defined as

$$
A(Z, L) \equiv f_{0}\left[\left(Z-Z_{c}\right)\left(L / L_{0}\right)^{c_{1}}\right]+a\left(L / L_{0}\right)^{-c_{3}},
$$

and the second data are defined as

$$
A(Z, L) \equiv f_{0}\left[\left(Z-Z_{c}\right)\left(L / L_{0}\right)^{c_{1}}\right]\left(1+\frac{a\left(L / L_{0}\right)^{-c_{3}}}{f_{0}[0]}\right),
$$

where $f_{0}[x]=\frac{1}{2}(\tanh x+1), t \equiv\left(Z-Z_{c}\right)$ is a relevant scaling variable, $L$ is the system size. Both forms obey a finite-size scaling law, and the $L^{-c_{3}}$ term is the correction to scaling. In particular, both forms are equal to the first-order of the Taylor expansion of Eq. (13): $f_{0}$ is shared, but $f_{1}$ is constant or proportional to $f_{0}$, respectively. We choose the values of the critical exponents close to those of the three-dimensional Ising model; we set the values of parameters as $Z_{c}=0, c_{1}=1.5$, $c_{3}=0.8, a=0.01$, and $L_{0}=48$. We generate an example data set of $A$ by adding a Gaussian noise with mean 0 and variance $(\delta A)^{2}$ to Eqs. (21) or (22). We consider four different system sizes: $L=8,12,16,24$. The number of data points for each system size is 17 . Because of the existence of the correction term, the crossing point between different system sizes shifts from the critical point [see the inset of Fig. 1(a)]. The minimum size of the correction term is about $4 \%$ of the thermodynamic scaling function at the critical point $Z=Z_{c}$.

Using kernel methods, we infer $Z_{c}$ and $c_{1}$ from a data set of $A$ with a precision $\delta A$. We start from correct values of physical parameters $\left(Z_{c}, c_{1}, c_{2}, c_{3}\right)$ for the convergence of nonlinear optimization process in the first stage of our kernel 

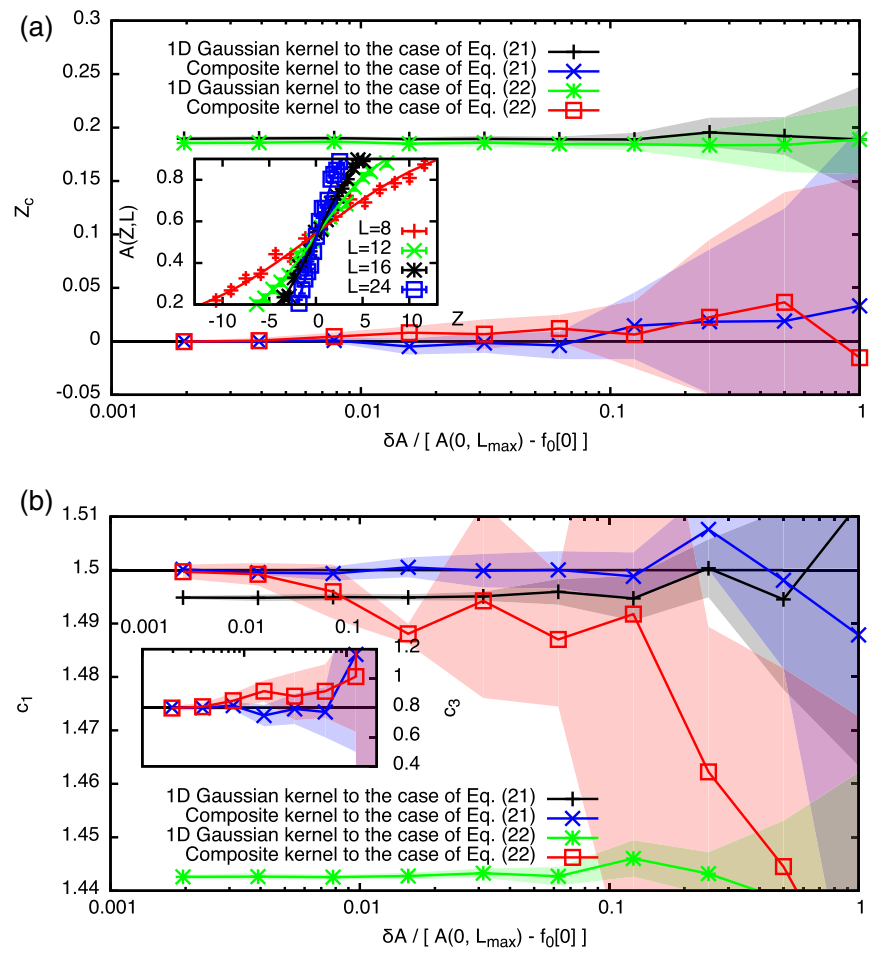

FIG. 1. (Color online) Average of inferred (a) $Z_{c}$, (b) $c_{1}$, and [inset of (b)] $c_{3}$ for ten data sets of $A$ with a precision $\delta A$. The shaded band denotes the variance of inferred results. Horizontal solid lines show correct values for each. Inset of (a): an example data set of $A$ obtained by using Eq. (21).

method. Here we fix $c_{2}$ as zero, because $A$ in Eqs. (21) or (22) is dimensionless. Because of the normalization of rescaled variables, we can safely start from 1 for all hyperparameters $\left(\theta_{2}, \theta_{i, 0}, \theta_{i, 1}\right)$. Starting from the result of the first optimization step, we estimate averages and confidential intervals of parameters by 1000 hybrid Monte Carlo samples.

Figures 1(a) and 1(b) show inference results for $Z_{c}$ and $c_{1}$ as a function of precision $\delta A$, respectively. We average out results of ten data sets. We call the original kernel in Ref. [12] as the one-dimensional (1D) Gaussian kernel; it is equal to $M=0$ in Eq. (14). Whereas inferences from the 1D Gaussian kernel quickly converge to incorrect values, those from the composite kernel effectively converge to the correct ones. However, it is necessary for the data precision to be within $10 \%$ for the size of the correction term. The case of Eq. (22) may be harder than that of Eq. (21), because the deviation from the correct value of $c_{1}$ from the 1D Gaussian kernel is larger. However, the composite kernel succeeds in its inference from high-precision data in both cases without knowledge of the correction form. We notice that the composite kernel has the same performance as the 1D Gaussian kernel in the case of no correction to scaling. In general, it is difficult to infer the value of the irrelevant scaling exponent with precision. However, when the data precision is improved, the result of the inference should converge to a correct value. The inset of Fig. 1(b) shows inference results of irrelevant scaling exponents $c_{3}$ obtained by using the composite kernel. Although the variances of the inferred $c_{3}$ values are large in both cases, they effectively are improved by the data precision. Finally, the inference results converge to a correct value.

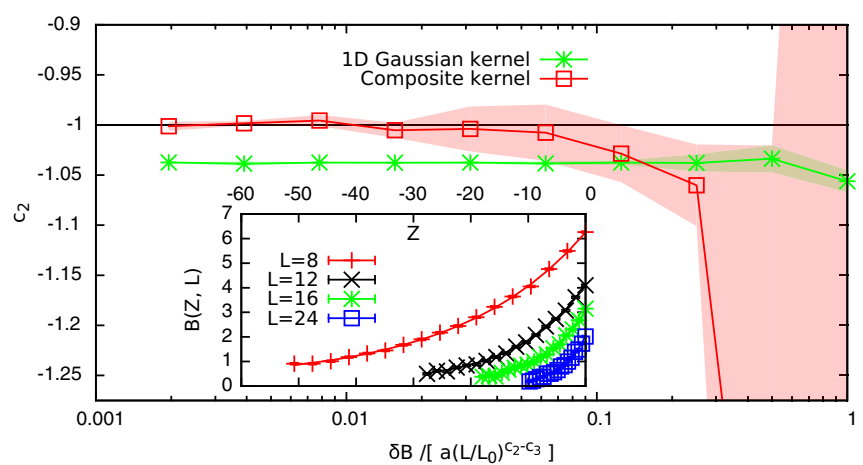

FIG. 2. (Color online) Average of inferred $c_{2}$ for ten data sets of $B$ with a precision $\delta B$. The shaded band denotes the variance of inferred results. The horizontal line shows the correct value of $B$. Inset: an example data set of $B$.

\section{General observable}

In the case of a general observable, we also need to infer $c_{2}$ in the scaling law of Eq. (1). We test the case of a general observable defined as

$$
\frac{B(Z, L)}{\left(\frac{L}{L_{0}}\right)^{c_{2}}} \equiv g_{0}\left[\left(Z_{c}-Z\right)\left(\frac{L}{L_{0}}\right)^{c_{1}}\right]+a\left(\frac{L}{L_{0}}\right)^{-c_{3}},
$$

where $g_{0}(x)=\exp \left(-c_{2} x / c_{1}\right)$ for $Z \leqslant Z_{c}$. Here, we set $c_{2}$ as -1 , and the values of other parameters are equal to those in the dimensionless case of $A$. Figure 2 shows the average of inference results of $c_{2}$ as a function of precision $\delta B$. We average out results of ten data sets of $B$. The composite kernel succeeds in the inference of $c_{2}$ when the precision is within $10 \%$ for the size of the correction term. However, the 1D Gaussian kernel method always converges to incorrect values, because it does not consider the existence of a correction to scaling. We notice that the performances for $Z_{c}, c_{1}$, and $c_{3}$ are similar to those in the dimensionless case.

\section{E. Ising model on cubic lattices}

Last, we apply kernel methods to the scaling analysis of the Ising model on cubic lattices. The Hamiltonian is written as $H \equiv-J \sum_{\langle i j\rangle} S_{i} S_{j}$, where the spin variables are $S_{i}= \pm 1$, and $\langle i j\rangle$ denotes a pair of nearest neighboring sites on a cubic lattice. The finite-temperature phase transition defines the three-dimensional Ising universality class, which widely covers a variety of critical systems. However, to confirm the universality class precisely, we have to take into account corrections to scaling in the scaling analysis [22]. Fortunately, we can obtain high precision data by using a sophisticated Monte Carlo algorithm for this model. Thus, it is a good example for the scaling analysis with a correction to scaling.

\section{Binder ratio of Ising model on cubic lattices}

We calculated Binder ratio, $\left\langle\left(\sum_{i} S_{i}\right)^{4}\right\rangle /\left[\left\langle\left(\sum_{i} S_{i}\right)^{2}\right\rangle\right]^{2}$, from $L=4$ to 32 by using the cluster algorithm with an improved estimator [23]. The simulation code is based on the ALPS library [24]. The number of temperature values for each system size is 17 . To ensure high precision, we took about $10^{8}$ samples for each point. 
(a)
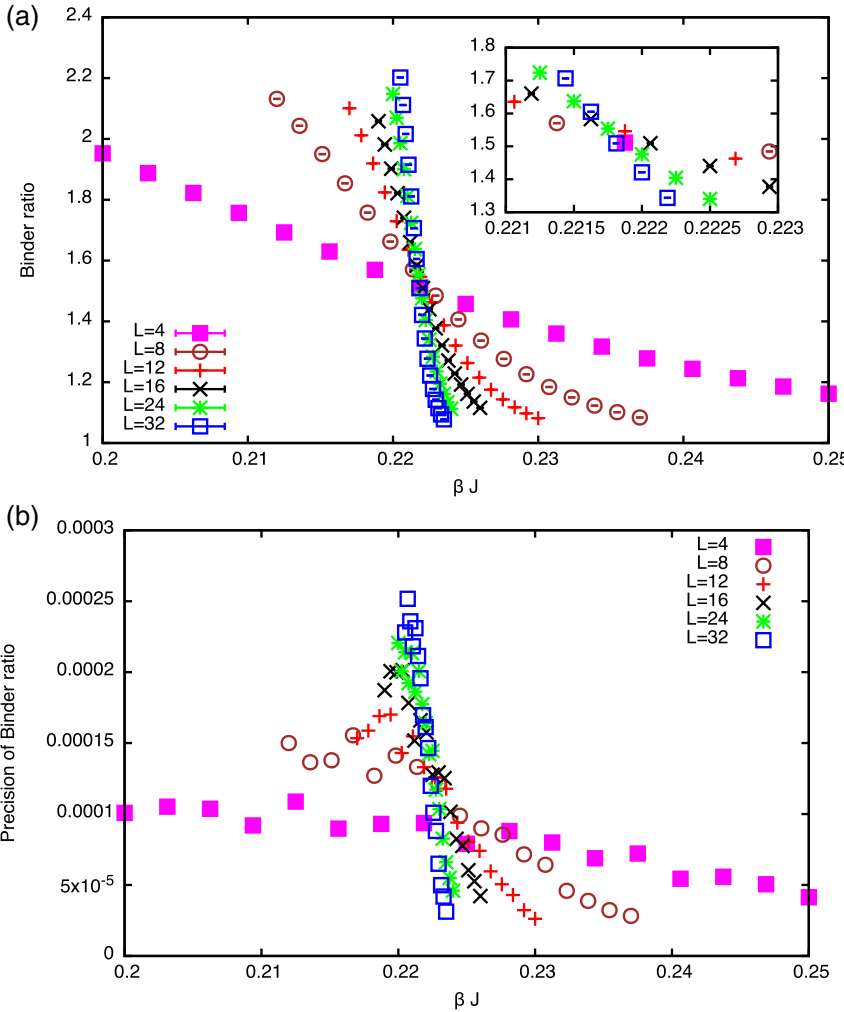

FIG. 3. (Color online) (a) Binder ratio of Ising model on cubic lattices. Inset: Binder ratio near a critical point. (b) Precision of Binder ratio in Monte Carlo calculations.

Figure 3(a) shows Binder ratios from $L=4$ to 32. In the thermodynamic limit, Binder ratios have a single crossing point at a critical temperature. However, due to a correction to scaling, there is a shift of crossing point in the finite system sizes. As shown in Fig. 3(a), Binder ratios almost share a single crossing point. The inset of Fig. 3(a) shows Binder ratios near the critical point (in Ref. [22], $\left.\beta_{c} J=0.22165463(8)\right]$. The crossing point between neighboring system sizes shifts, indicating the existence of corrections to scaling. Figure 3(b) shows the precision of data in Monte Carlo calculations. Because of critical slowing down, the precision becomes worse near a critical point. But, it may be enough to do a scaling analysis with a correction to scaling.

\section{Inference results}

We applied two kernel methods to the Binder ratios on selected system sizes as a sequence of $N_{\text {set }}$ systems up to $L_{\max }$. The range of Binder ratios is [1.1,2.2]. In addition, we start from results of physical parameters in Ref. [22] for the nonlinear optimization in the kernel method. Figure 4(a) shows the inference results of an inverse critical temperature $\beta_{c} J$ and a critical exponent $1 / v$ as a function of $L_{\max }$. The $\beta_{c} J$ results obtained by using the 1D Gaussian kernel $[M=0$ in Eq. (14)], in which a correction to scaling is not assumed, slowly converge when $L_{\max }$ increases. In contrast, results obtained by using the composite $\operatorname{kernel}[M=1$ in Eq. (14)] quickly converge. For critical exponents $1 / v\left(=c_{1}\right)$ and $\omega\left(=c_{3}\right)$, we observe a similar behavior in Fig. 4(b) and the inset. If the number of $N_{\text {set }}$ increases, the ignorance of a correction to


FIG. 4. (Color online) Inference results of (a) $\beta_{c} J$ and (b) $1 / v$ from Binder ratios of the Ising model on cubic lattices. Inset of (b): Inference results of $\omega . N_{\text {set }}$ is the number of systems in a data group to which kernel methods are applied. $L_{\max }$ is the largest system size in a data group. Horizontal solid lines show the values of $\beta_{c} J, 1 / \nu$, and $\omega$ in Ref. [22]. The shaded bands show the confidential intervals of inference results.

scaling affects an inference result. In fact, the convergence of $N_{\text {set }}=5$ is slower than that of $N_{\text {set }}=4$ in both kernel methods. The $\beta_{c} J, 1 / \nu$, and $\omega$ results obtained by using the composite kernel for $N_{\text {set }}=4$ and $L_{\max }=32$ are 0.221652(2), 1.587(2), and $0.83(9)$, respectively. They are consistent with reported values in Ref. [22] of $\beta_{c} J=0.22165463(8), 1 / v=1.5873(6)$, and $\omega=0.832(6)$, which were estimated from the fine-tuned Blume-Capel model up to $L=360$. Figure 5 shows the

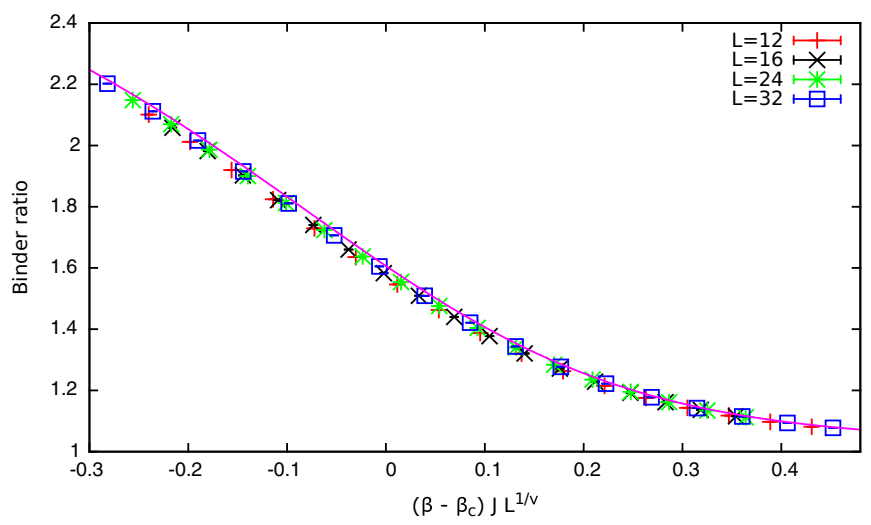

FIG. 5. (Color online) Finite-size scaling plot of Binder ratios with a correction to scaling. The values of $\beta_{c} J$ and $1 / v$ are 0.221652 and 1.587 , respectively. Solid pink line shows the thermodynamic scaling function inferred by the composite kernel. 
finite-size scaling plot when we apply the composite kernel method to the data set of $L=12,16,24$, and $32\left(N_{\text {set }}=4\right.$ and $L_{\max }=32$ ). Solid pink line shows the thermodynamic scaling function inferred by the composite kernel. It is just $\mu\left[\left(X_{1}, X_{2}=0\right)\right]$ in Eq. (11). The size of the correction to scaling can be roughly estimated from the inferred scaling function and data. In this case, it is about $1 \%$ of the scaling function near the critical point, and the data precision is within $2 \%$ for it. Therefore, if the data precision is high enough, the composite kernel can succeed in a scaling analysis of real data with a correction to scaling.

\section{SUMMARY}

In this work, we proposed the composite kernel method for a scaling analysis with corrections to scaling. This kernel has no ill-posedness in the inference problem for the scaling analysis. The key to the new kernel is the separation of relevant and irrelevant variables in the inference problem. It is based on the Taylor expansion by an irrelevant variable. We introduce the explicit form of corrections as a polynomial of irrelevant variables. In contrast, we do not need the explicit form of the scaling function related to the relevant variables. Therefore, the composite kernel method can be widely applied to real data in critical phenomena. We tested the performance of the new kernel method for example data sets that have corrections to scaling: three types of artificial data and a real data set of the Ising model on cubic lattices. The new kernel succeeded in the scaling analysis for all cases. In addition, we found that the data precision is important for successful scaling analysis. If the data precision is low, we cannot statistically distinguish a correction to scaling from the data noise. A precision within $10 \%$ for the correction term is necessary for succeeding in the scaling analysis by using the composite kernel method. Testing for a variety of critical phenomena requires further studies.

The goodness of fit is a useful test in the least-squares method. In the case of the kernel method, the regression function is nonlinear for inferred parameters. Thus, we cannot define a useful quantity similar to the $\chi^{2}$. However, to be exact, the regression function of the least-squares method is nonlinear for the inferred physical parameters as $\left(T_{c}, c_{1}, c_{2}, c_{3}\right)$. Thus, a simple $\chi$-square test does not cover the check of their inferences. In principle, the Bayesian framework gives us the distribution of inferred parameters as a marginal likelihood. We can compare the goodness of fit by the marginal likelihood. For example, we did not discuss the cutoff of the Taylor expansion in Eq. (13). To select a suitable cutoff, we can use the comparison of marginal likelihoods. However, we need additional calculations of them with addition of high precision for a high-order correction term. The simple procedure to check the goodness of fit remains in the future study.

\section{ACKNOWLEDGMENTS}

The author thanks Keith Slevin for enlightening discussions of the least-squares method. The author also thanks Naoki Kawashima for fruitful discussions. The present work is financially supported by JSPS KAKENHI, Grant No. 26400392, Japan.
[1] J. L. Cardy (ed.), Finite-Size Scaling (Elsevier, New York, 1988).

[2] T. Senthil, L. Balents, A. Vishwanath, S. Sachdev, and M. Fisher, Science 303, 1490 (2004).

[3] A. W. Sandvik, Phys. Rev. Lett. 98, 227202 (2007).

[4] A. W. Sandvik, Phys. Rev. Lett. 104, 177201 (2010).

[5] R. K. Kaul and A. W. Sandvik, Phys. Rev. Lett. 108, 137201 (2012).

[6] K. Chen, Y. Huang, Y. Deng, A. B. Kuklov, N. V. Prokof'ev, and B. V. Svistunov, Phys. Rev. Lett. 110, 185701 (2013).

[7] S. Pujari, K. Damle, and F. Alet, Phys. Rev. Lett. 111, 087203 (2013).

[8] M. S. Block, R. G. Melko, and R. K. Kaul, Phys. Rev. Lett. 111, 137202 (2013).

[9] K. Harada, T. Suzuki, T. Okubo, H. Matsuo, J. Lou, H. Watanabe, S. Todo, and N. Kawashima, Phys. Rev. B 88, 220408(R) (2013).

[10] T. Suzuki, K. Harada, H. Matsuo, S. Todo, and N. Kawashima, Phys. Rev. B 91, 094414 (2015).

[11] K. Slevin and T. Ohtsuki, Phys. Rev. Lett. 82, 382 (1999).

[12] K. Harada, Phys. Rev. E 84, 056704 (2011).

[13] T. Obuchi and H. Kawamura, Phys. Rev. B 87, 174438 (2013).

[14] Y. Motoyama and S. Todo, Phys. Rev. E 87, 021301 (2013).

[15] Y. Tomita, Phys. Rev. E 90, 032109 (2014).
[16] T. Nakamura and T. Shirakura, J. Phys. Soc. Jpn. 84, 013701 (2015).

[17] S. Takabe and K. Hukushima, Phys. Rev. E 89, 062139 (2014).

[18] T. Takahashi and K. Hukushima, Phys. Rev. E 91, 020102 (2015).

[19] T. Okubo, K. Oshikawa, H. Watanabe, and N. Kawashima, Phys. Rev. B 91, 174417 (2015).

[20] R. M. Neal, in Handbook of Markov Chain Monte Carlo (CRC Press, Boca Raton, FL, 2011), Chap. 5.

[21] The reference code of our kernel method for the scaling analysis with or without a correction to scaling is available from http://kenjiharada.github.io/BSA/.

[22] M. Hasenbusch, Phys. Rev. B 82, 174433 (2010).

[23] R. H. Swendsen and J.-S. Wang, Phys. Rev. Lett. 58, 86 (1987).

[24] B. Bauer, L. D. Carr, H. G. Evertz, A. Feiguin, J. Freire, S. Fuchs, L. Gamper, J. Gukelberger, E. Gull, S. Guertler, A. Hehn, R. Igarashi, S. Isakov, D. Koop, P. N. Ma, P. Mates, H. Matsuo, O. Parcollet, G. Pawłowski, J. D. Picon, L. Pollet, E. Santos, V. W. Scarola, U. Schollwöck, C. Silva, B. Surer, S. Todo, S. Trebst, M. Troyer, M. L. Wall, P. Werner, and S. Wessel, J. Stat. Mech.: Theory Exp. (2011) P05001. 